\title{
Urgency and emergency treatments in cardiovascular surgery during the COVID pandemic: results of extremized HUB and spoke organization in northern Italy.
}

\author{
Matteo Saccocci ${ }^{1}$, LUCA ATTISANI ${ }^{1}$, FRANCESCO FERRARO ${ }^{1}$, alessandro fossati ${ }^{1}$, \\ Emmanuel Villa ${ }^{1}$, Matteo Pegorer ${ }^{1}$, Antonio Messina ${ }^{1}$, Luca Luzzani ${ }^{1}$, Marco Cirillo ${ }^{1}$, \\ Stefania Blasi ${ }^{1}$, Zean Mhagna ${ }^{1}$, Luisa Cossu ${ }^{1}$, Margherita Dalla Tomba ${ }^{1}$, Stefano Pirrelli ${ }^{2}$, \\ Manfredo Rambaldini ${ }^{2}$, federico martinelli ${ }^{2}$, franco briolini ${ }^{3}$, Maurizio Merlo ${ }^{3}$, roberto \\ mezzetti $^{4}$, Vittorio Baratta ${ }^{5}$, Piergiorgio Sala ${ }^{6}$, Gabriele Piffaretti ${ }^{7}$, Paolo Panisi ${ }^{8}$, Stefano \\ Benussi $^{9}$, Claudio Muneretto ${ }^{9}$, raffaello bellosta ${ }^{1}$, and Giovanni Troise ${ }^{1}$ \\ ${ }^{1}$ Poliambulanza Foundation Hospitals \\ ${ }^{2}$ Azienda Socio Sanitaria Territoriale di Mantova \\ ${ }^{3}$ Azienda Ospedaliera Papa Giovanni XXIII \\ ${ }^{4}$ Policlinico San Marco \\ ${ }^{5}$ Azienda Sanitaria Locale Provincia di Cremona \\ ${ }^{6}$ Istituto Clinico S Anna \\ ${ }^{7}$ University of Insubria Faculty of Medicine and Surgery \\ ${ }^{8}$ Humanitas Gavazzeni \\ ${ }^{9}$ Università degli Studi di Brescia
}

June 28, 2020

\begin{abstract}
OBJECTIVES: Feasibility and results of cardiovascular hub-spoke networks to face COVID19 pandemic. The COVID-19 pandemic in Italy had the primary outbreak in the northern part of the country forcing the regional health care system to expand the availability of beds in the wards and intensive care units and to institute a Hub and Spoke hospital network to ensure assistance continuity for urgencies and emergencies. We report a descriptive analysis of the activity of the first 30 days of the Hub center. METHODS: Role of our Hub center was to guarantee 24/24h $7 / 7$ days cardiovascular surgical care for an area of 3.145.312 inhabitants' area. Hub-spoke reorganization permitted a significant increase of ICU and ward beds availability for COVID patients needing hospitalization in all peripheral centers. Records of all consecutive patients admitted were collected and analyzed. RESULTS: a total of 100 patients were evaluated in the study period . Hub and spoke cooperation have been successful, all patients affected by cardiovascular urgencies or emergencies found a highly specialized hospital and was evaluated and treated. Global reduction of elective and non-deferrable interventions in spoke centers was achieved for both vascular and cardiac surgery while we detected a significant increase of urgent vascular interventions for acute limb ischemia. We did not observe an increase of in-hospital mortality in non-infected patients. CONCLUSION: Hub and spoke network for cardiovascular pathology is an effective way to face healthcare needs during the pandemic.
\end{abstract}

\section{Hosted file}

manuscript.pdf available at https://authorea.com/users/337287/articles/462912-urgency-andemergency-treatments-in-cardiovascular-surgery-during-the-covid-pandemic-results-of- 
extremized-hub-and-spoke-organization-in-northern-italy

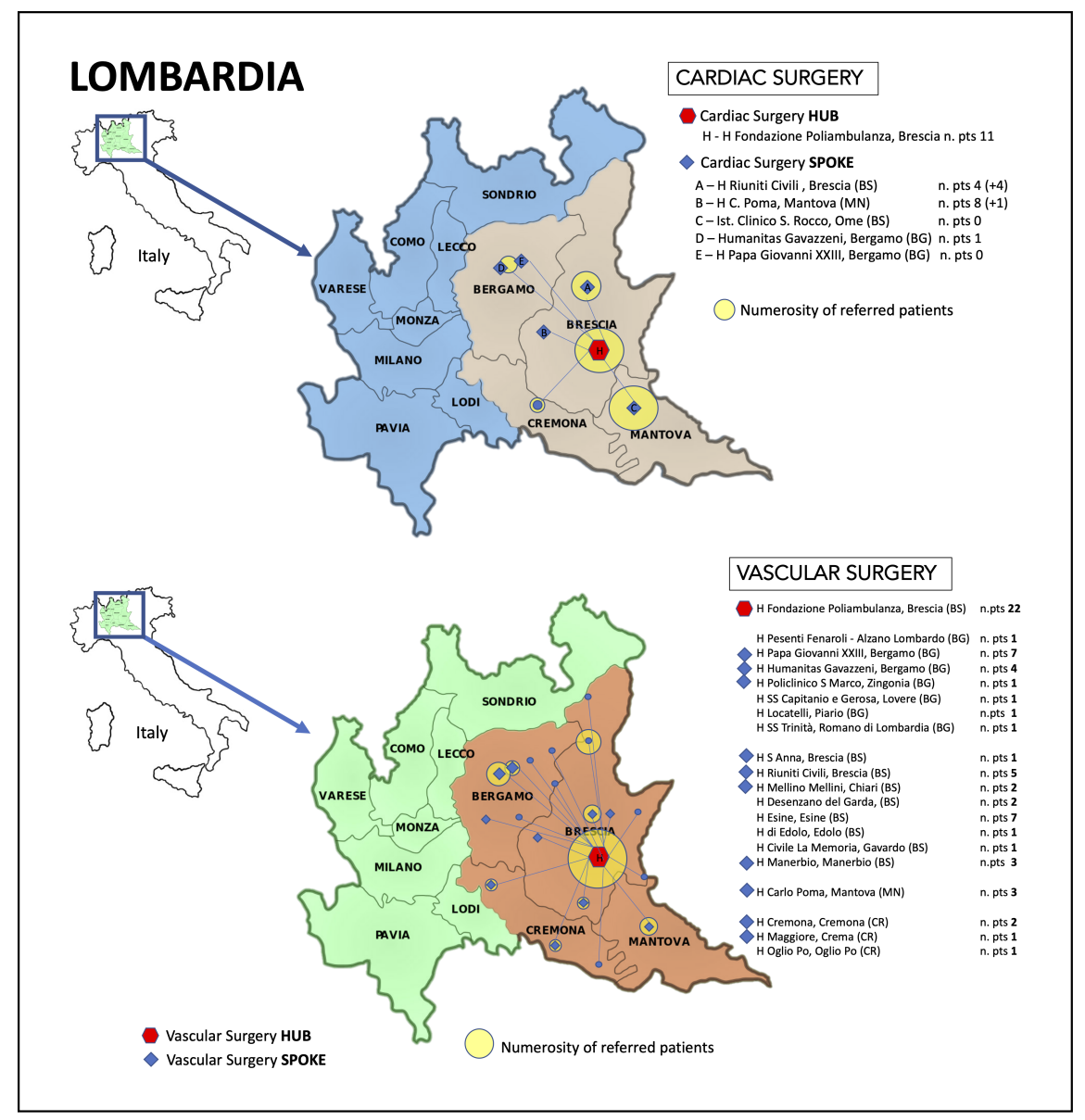

\section{Hosted file}

TABLES JCS.docx available at https://authorea.com/users/337287/articles/462912-urgency-andemergency-treatments-in-cardiovascular-surgery-during-the-covid-pandemic-results-ofextremized-hub-and-spoke-organization-in-northern-italy 